\title{
Más allá del efecto y de la intención: la proporcionalidad en los arbitrajes de inversiones
}

\author{
Beyond Effect and Intention: Proportionality in Investment Arbitration
}

\author{
Sebastián López Escarcena \\ ORCID: 0000-0002-0001-9989 \\ Pontificia Universidad Católica de Chile \\ Correo: rlopeze@uc.cl \\ Recibido: 10/07/2020 \\ Aceptado: 03/11/2020
}

Resumen: La forma de expropiación que prevalece hoy es la indirecta, cuya determinación constituye uno de los principales problemas a resolver en el derecho internacional de la inversión. La doctrina y jurisprudencia han identificado dos posibles soluciones centradas, respectivamente, en el efecto o en la intención de las medidas estatales. En el último tiempo, ha aparecido una tercera alternativa que cuenta con un aparente apoyo en ciertos tratados de inversión de nueva generación. Esta proviene de la jurisprudencia de la Corte Europea de Derechos Humanos relativa a la protección de la propiedad, y consiste en la aplicación del principio de proporcionalidad para determinar la naturaleza expropiatoria de las medidas judicialmente impugnadas por inversionistas extranjeros en arbitrajes internacionales. Esta posible solución, sin embargo, también presenta algunas dificultades de implementación. El propósito de este trabajo es estudiar la aplicación de la proporcionalidad en dichos arbitrajes. A través del análisis de la jurisprudencia relevante, y la utilización de los métodos dogmático y comparativo, se examina la aparición de este principio en el derecho internacional de la inversión, destacando las diferencias en su aplicación en los foros en los que se la ha utilizado, para concluir que este solo podrá constituir un instrumento útil para solucionar demandas de expropiación indirecta en la medida que cuente con una clara base normativa, que le permita ser empleado, en conjunto con el margen de apreciación, para determinar la legalidad de las medidas respectivas, no su naturaleza expropiatoria.

Palabras clave: inversión extranjera; tratados; expropiación indirecta; proporcionalidad; derechos humanos.

\begin{abstract}
Indirect expropriation is the prevailing form of expropriation today, and its determination constitutes one of the main problems in international investment law. Doctrine and case-law has identified two possible solutions centered, respectively, in the effects or in the intention of the measures adopted by the host state. Of late, a third alternative has appeared that finds possible support in certain last-generation investment treaties. Deriving from the case-law of the European Court of Human Rights on the protection of property, it applies the principle of proportionality to determine the expropriatory nature of those measures judicially challenged by foreign investors in international arbitrations. This possible solution, however, also presents some issues of implementation. The purpose of this work is to study the application of proportionality in these disputes. Through the analysis of the relevant case-law, and the use of the dogmatic and comparative methods, the present article examines the emergence of this principle in international investment law, highlighting the differences in its application in the fora in which it has been used, to conclude that in order to become a useful instrument for solving claims of indirect expropriation it must have a clear normative basis, allowing for its employment, in tandem with the margin of appreciation, to determine the lawfulness of the respective measures, not their expropriatory nature.
\end{abstract}

Keywords: foreign investment; treaties; indirect expropriation; proportionality; human rights. 


\section{Introducción}

Para promover la inversión extranjera hay que protegerla. Esto es justamente lo que hace la extensa red de tratados que hoy la regulan, al ofrecer ciertos derechos para los nacionales de los Estados parte de dichos acuerdos internacionales, que están garantizados por un mecanismo de solución de controversias entre inversionistas extranjeros y Estados anfitriones. Tales derechos incluyen la expropiación condicionada; el trato justo y equitativo; la protección y seguridad plenas; y el trato nacional y de nación más favorecida; entre otros. ${ }^{1}$

Hacia fines del año 2019, alrededor de 3.300 acuerdos internacionales de inversión habían sido celebrados, la mayoría de ellos después de 1990. Más de 2.600 de dichos tratados están en vigencia. De estos, unos 2.900 son tratados bilaterales de inversión (TBI), y aproximadamente 390 son acuerdos de integración económica (AIE), tanto multilaterales como bilaterales, que incluyen un compromiso de promover y proteger la inversión extranjera (UNCTAD, 2020b, p. 106). ${ }^{2}$

Ejemplos de estos son el Acuerdo de Promoción y Protección de las Inversiones de la Asociación de Naciones del Sudeste Asiático (ANSA) de 1987³; el Capítulo 11 del Tratado de Libre Comercio de América del Norte (TLCAN) de 1992 ${ }^{4}$; y las Partes III y V del Tratado sobre la Carta de la Energía (TCE) de $1994^{5}$. Dentro de los más recientes, se puede nombrar el Capítulo 10 del Acuerdo de Libre Comercio entre Centroamérica, EE.UU. y la República Dominicana de 2004, mejor conocido como CAFTA ${ }^{6}$; el Capítulo 8 del Acuerdo Comprehensivo Económico y Comercial entre Canadá y la Unión Europea

\footnotetext{
${ }^{1}$ Sobre los tratados de inversión ver en general Dolzer \& Stevens (1995), Salacuse (2015), Vandevelde (2010) y Yannaca-Small (2018).

${ }^{2}$ En la actualidad, casi 2.400 TBI han entrado en vigor, mientras que aproximadamente 320 AIE están vigentes. Los tratados de inversión están disponibles en https://investmentpolicyhub.unctad.org/IIA

${ }^{3}$ En vigor desde 1990, y modificado por un protocolo en 1996.

${ }^{4}$ En vigor desde 1994. En 2018, Canadá, EE.UU. y México acordaron reemplazar el TLCAN por el Tratado entre México, EE.UU. y Canadá o T-MEC, también llamado USMCA (United States-Mexico-Canada Agreement). El TLCAN se mantuvo en vigencia hasta que sus miembros ratificaron el T-MEC. En este nuevo tratado, el capítulo de inversión es el 14, el cual restringe los arbitrajes de inversiones a EE.UU. y México, únicamente para casos de incumplimiento de las cláusulas de trato nacional y de trato de nación más favorecida, y de expropiación directa, no más allá de cuatro años después de que el demandante haya tomado conocimiento de la violación convencional.

${ }^{5}$ Vigente desde 1998.

${ }^{6}$ Sigla proveniente de su nombre en inglés: Free Trade Agreement between Central America, the Dominican Republic and the United States of America. El CAFTA está en vigencia desde el 2009.
} 
de 2016, o CETA7 ${ }^{7}$ el Protocolo de Cooperación y Facilitación de Inversiones IntraMERCOSUR del 2017; y el Capítulo 9 del Acuerdo Integral y Progresivo para la Asociación Transpacífico del 2018, o CPTPP ${ }^{9}$. Las normas de inversión extranjera en estos AIE son bastante similares a las de los TBI.

Que el Estado tenga un derecho a expropiar las inversiones extranjeras es algo que no se cuestiona hoy, ni consuetudinaria ni convencionalmente. La amplia red de tratados de inversión que existe en la actualidad incorpora esta facultad estatal, condicionando su ejercicio al cumplimiento de cuatro requisitos: que la medida expropiatoria sea adoptada por causa de utilidad pública; en forma no-discriminatoria; que vaya acompañada del pago de una indemnización pronta, adecuada y efectiva; y se apegue en todo momento al debido proceso (Mouyal, 2018, pp. 164-166). ${ }^{10}$

Aun cuando no hay un concepto de expropiación aceptado transversalmente en el derecho internacional, se la puede definir como la privación real o efectiva de la propiedad, sea que despoje al dueño de su bien, reclamando su título, o que destruya o afecte gravemente su utilidad. Esta noción incluye las formas tanto directa como indirecta de expropiar (Mouyal, 2018, pp. 160-161, 166-169; Stern, 2008, pp. 34-37). De estas, la prevaleciente en el derecho internacional de la inversión es, sin lugar a duda, la expropiación indirecta. ${ }^{11}$

La gran pregunta que plantea esta modalidad de privación de propiedad es: ¿en qué momento pasa una regulación estatal a ser expropiatoria, e indemnizable como tal? (Kriebaum, 2007, pp. 718-719, 722; Perkams, 2010, p. 108; Stern, 2008, p. 29). ${ }^{12}$ Esta es una duda que puede presentarse cada vez que un Estado adopte un amplio abanico de medidas, como por ejemplo ambientales, laborales, de libre competencia, de planificación urbana, de protección al consumidor, y tributarias, entre otras. Si bien no hay una respuesta tipo que se anticipe a las múltiples circunstancias en las que esta interrogante

\footnotetext{
${ }^{7}$ Por su sigla en inglés, derivada de EU-Canada Comprehensive Economic and Trade Agreement. El CETA todavía no ha entrado en vigor. Este tratado contiene interesantes innovaciones en su mecanismo de solución de controversias. Ver en general Von Walter \& Andrisani (2019).

${ }^{8}$ Aún no está en vigencia.

${ }^{9}$ Sigla en inglés de Comprehensive and Progressive Agreement for Trans-Pacific Partnership. Los estadosparte de este tratado, vigente desde el 2018, son Australia, Brunei, Canadá, Chile, Japón, Malasia, México, Nueva Zelanda, Perú, Singapur y Vietnam. Para Canadá y México, este tratado viene a reemplazar al antiguo TLCAN, en lo que respecta a la inversión extranjera.

${ }^{10}$ Ver Kriebaum (2007, pp. 719-720).

${ }^{11}$ Las expropiaciones directas ya no se presentan con la frecuencia que lo hacían (Mouyal, 2018, pp. 161, 167, 168; Ranjan \& Anand, 2016, p. 130). Ver Kriebaum (2007, p. 717) y Stern (2008, p. 29, 37-38).

${ }^{12}$ Ver Ranjan \& Anand (2016, p. 130-131).
} 
puede manifestarse, la doctrina y la jurisprudencia internacional han identificado ciertas vías de solución. Por un lado, están las doctrinas del efecto y del poder de policía, que tienden a favorecer los intereses de los inversionistas extranjeros o de los Estados anfitriones, respectivamente. Por otro, está la aplicación del principio de proporcionalidad a la protección contra la expropiación ilegal, como posible alternativa conciliadora de ambos intereses.

El artículo que sigue, estudia esta tercera posibilidad de solución. Sus secciones primera y segunda explican por qué algunos Estados han comenzado a reemplazar sus tratados de inversión, y que implicancia tiene esto para la protección contra la expropiación ilegal que estos contienen. Las secciones tercera y cuarta de este artículo, en tanto, analizan la aparición y posterior reiteración del principio de proporcionalidad en la jurisprudencia de los arbitrajes de inversiones. En su quinta sección, el trabajo termina por destacar las diferencias que presenta la implementación de la proporcionalidad en los distintos foros en los que se le ha utilizado, dilucidando cuál de estas se condice mejor con la finalidad última de dicho principio.

\section{Tratados de inversión}

Hasta hace unos pocos años, existían dos modelos principales de tratado de inversión, el europeo y el estadounidense; y sus diferencias no eran mayores. Mientras el modelo de EE.UU. era más extenso y detallado, al incorporar disposiciones de los tratados de amistad, comercio y navegación (TACN), que antecedieron a los TBI por casi dos siglos, el de los distintos Estados de Europa era más breve y de una redacción más general, normalmente prescindiendo de lo dispuesto en los TACN (López Escarcena, 2014a, p. 113-117). ${ }^{13}$ Tanto un modelo como el otro incluían protecciones contra la expropiación ilegal, sea esta directa e indirecta. En otras palabras, conforme a estos acuerdos internacionales, el ejercicio de la facultad expropiatoria debía cumplir los cuatro requisitos de utilidad pública, no discriminación, indemnización pronta, adecuada y efectiva, y debido proceso. Al respecto, los tratados de inversión tampoco diferenciaban las expropiaciones de las nacionalizaciones, cuya relación es de género a especie. ${ }^{14}$

\footnotetext{
${ }^{13}$ En cuanto a los TACN y los TBI de EE.UU. ver en general Vandevelde (2009b; 2017).

${ }^{14}$ Esto, por cuanto la nacionalización es una expropiación de una o más industrias o recursos principales, dentro de un programa de reforma social y económica. Sobre las nacionalizaciones en el derecho internacional ver en general Ruzza (2017).
} 
Estos modelos se mantuvieron sin mayores cambios, por el tiempo que el Estado exportador de capital siguió siendo sinónimo de país desarrollado. Cuando los demandados en arbitrajes de inversiones dejaron de ser exclusivamente los Estados en vías de desarrollo, una nueva generación de tratados de inversión comenzó a reemplazar a dichos modelos inaugurales (Bücheler, 2015, pp. 136-141; Calamita, 2014, pp. 196198). ${ }^{15}$ El primero en tomar este camino fue EE.UU., cuya ley de promoción del comercio de 2002 estableció como uno de los principales objetivos de negociación de dicho estado, asegurar a los extranjeros protecciones que no excedan de las que gozan bajo el derecho estadounidense quienes invierten en ese país (López Escarcena, 2014a, pp. 121-123, 159161, 187-190, 199-200). Consecuentemente, su modelo de TBI de 2004 introdujo diversas modificaciones a las cláusulas de expropiación condicionada, a fin de reconducir su interpretación judicial hacia soluciones similares a las que ofrece la jurisprudencia estadounidense de las expropiaciones regulatorias (Vandevelde, 2009a). ${ }^{16}$

Por esos años, la doctrina de los países desarrollados empezó a manifestarse en contra del derecho internacional de la inversión, identificando diversos problemas en la extensa red de tratados que hasta entonces había apoyado entusiastamente. ${ }^{17}$ Esta reacción que comenzó a provocar el derecho internacional de la inversión, se centró en aspectos tanto sustantivos como adjetivos. ${ }^{18}$

No tuvo que pasar mucho tiempo para que estas críticas encontraran asidero en ciertos Estados tradicionalmente importadores de capital, como Bolivia, Ecuador y Venezuela. Además de retirarse del Convenio sobre Arreglo de Diferencias Relativas a Inversiones entre Estados y Nacionales de Otros Estados en 2008, 2009 y 2012, respectivamente, estos tres países empezaron a terminar algunos de los TBI que previamente habían celebrado con otros Estados. ${ }^{19}$ No fueron los únicos, pues Sud África e Indonesia también procedieron a poner término a ciertos TBI por esos años (Lusa

\footnotetext{
${ }^{15}$ Ver Kingsbury \& Schill (2010, p. 76), Mostafa (2008, pp. 270-271) y Vandevelde (2009a, pp. 288, 290, 293-294). Cf. de Nanteuil (2017, p. 17).

${ }^{16}$ El modelo de TBI estadounidense fue posteriormente reemplazado en 2012, sin mayores variaciones en lo que se refiere a sus cláusulas de expropiación condicionada.

${ }^{17}$ Previo a esto, solo los autores cercanos al nuevo orden económico internacional habían reparado en estos asuntos. Uno de ellos es Muthucumaraswamy Sornarajah, crítico de estos acuerdos internacionales desde antes que EE.UU. promulgara la ley de promoción del comercio y modificara su política de inversión extranjera (Lim, 2016). Respecto al nuevo orden económico internacional ver en general Sacerdoti (2015). ${ }^{18}$ Ver la Declaración Pública sobre el Régimen Internacional de Inversiones, hecha el 31 de agosto de 2010 por profesores de derecho de distintos países en la Universidad York, Canadá. Recuperado de https://www.osgoode.yorku.ca/public-statement-international-investment-regime-31-august-2010/ Ver en general Kulick (2018) y Waibel et al. (2010).

${ }^{19}$ Ver en general Durney (2013).
} 
Bordin, 2018, pp. 217-219). ${ }^{20}$ Como sea, las cifras disponibles demuestran que al menos uno de los cuestionamientos que se le ha hecho al derecho internacional de la inversión es infundado: que los tribunales internacionales en arbitrajes de inversiones fallan normalmente a favor de los inversionistas extranjeros, y en desmedro de los Estados anfitriones. En efecto, desde que se resolviera el primer arbitraje de inversiones en 1990, el número de este tipo de disputas públicamente conocidas ha superado en la actualidad las 1.000 (UNCTAD, 2020a, pp. 1-3; 2019, 2020b, pp. 110-112). ${ }^{21}$ De estas, 674 han sido resueltas por tribunales arbitrales internacionales.

A la fecha, 120 Estados han sido demandados en una o más arbitrajes de inversiones. ${ }^{22}$ Más de un tercio del total de los resueltos a la fecha, han sido decididos a favor del Estado, la mitad de los cuales por falta de jurisdicción del tribunal correspondiente; aproximadamente un cuarto del total de casos ha sido fallado en favor del inversionista extranjero; y otro cuarto del total han sido resueltos por acuerdo entre las partes. El resto de los casos fueron discontinuados, y unos pocos se resolvieron a favor del inversionista extranjero, pero sin llegar a establecerse daños. ${ }^{23}$

Inspirados en la experiencia estadounidense, Canadá y la Unión Europea han modificado su política de inversión extranjera. El modelo de tratado de inversión canadiense fue reemplazado en 2004, el mismo año que EE.UU. sustituyera su modelo de TBI (Fortier, 2009). En 2009, con la entrada en vigor del Tratado de Lisboa, la competencia para celebrar acuerdos internacionales que regulan la inversión extranjera pasó de los Estados miembros a la Unión Europea, la cual empezó a negociar textos convencionales que incluyen clarificaciones a las cláusulas de expropiación condicionada (Bücheler, 2015, p. 141; Titi, 2015). China, asimismo, ha introducido modificaciones a los capítulos de inversión de sus AIE, siguiendo el modelo estadounidense (Wei, 2015). Australia ha reformado sus tratados de inversión, incorporando las clarificaciones de los

\footnotetext{
${ }^{20}$ Ver Ranjan (2014, pp. 859-861).

${ }^{21}$ El primer arbitraje de inversiones que se falló es "Asian Agricultural Products c/ Sri Lanka" (1990). Las decisiones judiciales dictadas en este tipo de arbitrajes están generalmente disponibles en https://www.italaw.com/

22 Argentina es el país con el mayor número de disputas interpuestas en su contra: 62. En América Latina, le sigue Venezuela con 51. México y Ecuador vienen después con 33 y 24 casos, respectivamente. Antes de estos países, vienen España (52 casos), la República Checa (40), Egipto (37), Polonia (30) y Canadá (29). Respecto a las nacionalidades más frecuentes de los demandantes son: EE.UU. (183 casos), Países Bajos (111), Reino Unido (86), Alemania (69), España (57), Canadá y Francia (51, cada uno), etc.

${ }^{23}$ Para información actualizada a este respecto ver el Navegador de Solución de Disputas de Inversión de la Conferencia de la ONU sobre Comercio y Desarrollo, mejor conocida como UNCTAD por su sigla en inglés, recuperado de https://investmentpolicy.unctad.org/investment-dispute-settlement
} 
acuerdos internacionales de EE.UU. y Canadá a sus cláusulas de protección contra la expropiación ilegal (Bücheler, 2015, p. 138-139). ${ }^{24}$ En América Latina, lo habitual ha sido adaptar modelos convencionales provenientes de Europa. No obstante, aquí también hay novedades a este respecto. Colombia, por ejemplo, adoptó el modelo estadounidense en sus TBI y AIE (Duggal et al., 2019). La mayoría de estos tratados de inversión de última generación, tanto de origen americano como europeo, incluyen clarificaciones destinadas a guiar la interpretación y aplicación de sus disposiciones que hacen los tribunales internacionales en arbitrajes de inversiones. A la cláusula de la expropiación condicionada, en concreto, se la han incorporado elementos de derecho comparado para encauzar la solución jurisprudencial tradicionalmente dada a las demandas de expropiación indirecta, hacia una que se acerque a la que ofrecen los tribunales estadounidenses, que es el país de origen de estas clarificaciones.

\section{Las expropiaciones indirectas}

Como célebremente dijera Oliver Wendell Holmes, la propiedad puede ser regulada hasta cierto punto, pero si esta regulación llega demasiado lejos, tendrá que ser reconocida como una expropiación (Asteriti, 2013, p. 461; Bücheler, 2015, p. 124). ${ }^{25}$ Cuándo ocurre esto es la gran pregunta en el derecho internacional de la inversión. Este problema es ciertamente complejo, y solo se puede resolver caso a caso (Mouyal, 2018, p. 167). Si bien no hay respuesta tipo aplicable a cualquier situación regulatoria/expropiatoria que se presente, la doctrina y la jurisprudencia internacional han ofrecido ciertas alternativas de solución.

A la primera de estas se le conoce como doctrina del efecto, por cuanto centra su análisis en la consecuencia o resultado que la medida estatal tiene en la inversión extranjera. Para esta teoría, el límite entre regulación no indemnizable y expropiación indirecta no es otro que la privación de la propiedad afectada (Bücheler, 2015; De Nanteuil, 2015, pp. 10-11; Mouyal, 2018, pp. 187-189; Ranjan \& Anand, 2016, pp. 131132). ${ }^{26}$ A la segunda se le ha dado el nombre de doctrina del poder de policía o de la potestad regulatoria, y agrega un elemento esencial a tener en consideración al momento

\footnotetext{
${ }^{24}$ Este país incluso ha eliminado la posibilidad de iniciar arbitrajes de inversiones en sus nuevos tratados.

${ }^{25}$ Ver en general "Pennsylvania Coal. c/ Mahon" (1922). Recuperado de https://caselaw.findlaw.com/ussupreme-court/260/393.html

${ }^{26}$ Ver Henckels (2012, p. 225), Kingsbury \& Schill (2010, p. 90), Kriebaum (2007, pp. 724-725), Mostafa (2008, p. 268), Perkams (2010, p. 110-111) y Stern (2008, pp. 38-45).
} 
de hacer dicho análisis. Además del efecto de la medida en cuestión, esta teoría atiende a la intención del Estado y el contexto en el que fue adoptada (Bücheler, 2015, pp. 127129; De Nanteuil, 2017, pp. 10-11; Mouyal, 2018, pp. 176-178; Ranjan \& Anand, 2016, pp. 132-136). ${ }^{27}$ Ambas alternativas han sido criticadas por favorecer los intereses de los inversionistas extranjeros o de los Estados anfitriones, respectivamente, y por ofrecer una solución de todo o nada al difícil y siempre relevante problema de la expropiación indirecta.

El primer antecedente jurisprudencial de la doctrina del efecto puede encontrarse en el "Navieros Noruegos" (1922). ${ }^{28}$ Sin embargo, fue el Tribunal de Reclamaciones IránEE.UU. quien la desarrolló en decisiones judiciales como "Tippets" (1984) y otras, frecuentemente citadas en el derecho internacional de la inversión. ${ }^{29}$ Expresa o implícitamente apoyada por un número importante de autores, la teoría del efecto puede incluso apreciarse en algunos TBI o AIE. La jurisprudencia de arbitrajes de inversiones ha optado por esta aproximación en varias oportunidades (López Escarcena, 2014a, pp. 112-224). Su formulación más conocida es la de "Metalclad" (2000) (Asteriti, 2013, p. 458). ${ }^{30}$ La doctrina del poder de policía, en cambio, tiene como precedente remoto al caso "Oscar Chihn" (1934). ${ }^{31}$

Como su contraparte, esta teoría fue posteriormente desarrollada por el Tribunal de Reclamaciones Irán-EE.UU., pero solo excepcionalmente en "Sea-Land” (1984). ${ }^{32}$ Tal como fuera expuesta en "Methanex" (2005), la teoría del poder de policía ha tenido mejor suerte en los tribunales de arbitrajes de inversiones (Asteriti, 2013, p. 458-459). ${ }^{33}$ Más allá de esta jurisprudencia, es en los TBI y los capítulos de inversión de los AIE de nueva generación de ciertos Estados donde esta alternativa ha encontrado un verdadero sustento, fruto de circunstancias históricas concretas que bien conviene recordar.

\footnotetext{
${ }^{27}$ Ver Kriebaum (2007, pp. 725-727), Mostafa (2008, pp. 268, 272-274), Perkams (2010, pp. 110-111) y Stern (2008, pp. 45-50). Cf. Henckels (2012, p. 225) y Kingsbury \& Schill (2010, p. 90).

28 Ver en general "Navieros Noruegos (Noruega c/ EE.UU.)" (1922). Recuperado de https://legal.un.org/riaa/cases/vol_I/307-346.pdf

29 La jurisprudencia del Tribunal de Reclamaciones Irán-EE.UU. fue recuperada de https://www.iusct.net/Pages/Login.aspx. Sobre la jurisprudencia de este tribunal ver en general López Escarcena (2013).

${ }^{30}$ Ver "Metalclad c/ México" (2000, párr. 103).

31 Recuperado de https://www.icj-cij.org/files/permanent-court-of-internationaljustice/serie_AB/AB_63/01_Oscar_Chinn_Arret.pdf

${ }^{32}$ Recuperado de https://legal.un.org/riaa/cases/vol_I/307-346.pdf

${ }^{33}$ Ver "Methanex c/ EE.UU.", (2005, párr. 7).
} 
En 1992, Canadá, EE.UU. y México celebraron el TLCAN, cuyo Capítulo 11 se tituló "Inversión". Entonces, se partía de la base que los dos Estados desarrollados de este tratado iban a jugar el rol de país exportador de capital, mientras que el de país importador de capital iba a quedar en manos del único Estado en vías de desarrollo de este acuerdo internacional. En otras palabras, que los derechos de su Capítulo 11 iban a favorecer exclusivamente a los inversionistas canadienses y estadounidenses en México. Para sorpresa de todos, no pasó mucho tiempo para que los arbitrajes de inversiones fueran dirigidos también contra Canadá y EE.UU. Fue entonces que el diseño original de los tratados de inversión, destinados principalmente a proteger a los nacionales de los Estados exportadores de capital, comenzó a ser puesto en duda en dichos países. En ese contexto, EE.UU. promulgó en 2002 la ley de promoción del comercio, y en cumplimiento de esta sus tratados de inversión comenzaron a incluir ciertas clarificaciones destinadas a guiar la interpretación de estos acuerdos internacionales en los arbitrajes de inversiones hacia la teoría que más se asemeja a la doctrina de las expropiaciones regulatorias de su derecho interno: la del poder de policía. ${ }^{34}$

En efecto, de acuerdo con la jurisprudencia de los tribunales de EE.UU. deben recibir una justa indemnización la ocupación física permanente de la propiedad, o la destrucción de un $100 \%$ de su valor como resultado de una regulación. La doctrina de este país llama a estas situaciones "eminent domain" e "inverse condemnation", respectivamente. ${ }^{35} \mathrm{Si}$ no se presenta una o la otra, los tribunales tienen que realizar una investigación ad hoc para establecer si la medida regulatoria correspondiente es expropiatoria. En su investigación, los tribunales de EE.UU. deben guiarse por los factores identificados en el caso "Penn Central" (1978): el impacto de la regulación en el demandante; la extensión de su interferencia con expectativas específicas de inversión; y el carácter de la acción gubernamental (Asteriti, 2013, pp. 461-463). ${ }^{36}$ Esto explica que, desde hace casi dos décadas, los TBI y AIE de EE.UU. incluyan un anexo que incorpora

\footnotetext{
${ }^{34}$ De hecho, la expresión "police powers" que se utiliza en el derecho internacional de la expropiación procede del derecho constitucional estadounidense (Bücheler, 2015, pp. 128, 129). Cf. Asteriti (2013, pp. 456-457). Ver Ranjan \& Anand (2016, p. 133-134) y Vadi (2014b, p. 341; 2015, pp. 562-563). Ver en general Legarre (2007).

${ }^{35}$ En el primer caso, es la entidad estatal expropiante la que demanda, mientras que en el segundo, es el particular afectado por la medida quien lo hace.

${ }^{36}$ Ver en general "Penn Central Transportation Co. c/ Ciudad de Nueva York" (1978). Recuperado de https://caselaw.findlaw.com/us-supreme-court/438/104.html

Cabe destacar, que no es fácil obtener una indemnización por una medida expropiatoria en EE.UU. (Plater et al., 2010, pp. 891-932). Rara vez la jurisprudencia estadounidense ha resuelto que una regulación destruya el $100 \%$ del valor de la propiedad, o que se presenten los tres factores de "Penn Central".
} 
estos elementos a la protección contra la expropiación que proporciona el acuerdo internacional respectivo. Esto se puede ver, por ejemplo, en el Anexo 10-D del capítulo de inversiones del TLC Chile-EE.UU. (2003). ${ }^{37}$ Una clarificación similar puede encontrarse en los tratados de inversión de Canadá y de Colombia (Bücheler, 2015, pp. 137-138). El que utiliza actualmente la Unión Europea tiene algunas diferencias, pero en esencia plantea lo mismo (Bücheler, 2015, pp. 140-141).

\title{
"Tecmed" y la proporcionalidad europea
}

A las doctrinas del efecto y del poder de policía se ha venido a sumar una tercera posibilidad de solución para las demandas de expropiación indirecta en los arbitrajes de inversiones. Importada desde el derecho internacional de los derechos humanos, esta aproximación propone aplicar el principio de proporcionalidad al examen destinado a dilucidar el carácter expropiatorio de la medida regulatoria en cuestión. No es fácil encontrar una mención a este principio en los tratados de inversión (Calamita, 2014, pp. 167-168; Vadi, 2014a, pp. 207-208; 2014b, p. 349; 2015, p. 578) ${ }^{38}$ Más suerte ha tenido en la jurisprudencia de arbitrajes de inversiones, donde algunos tribunales lo han utilizado de manera confusa.

El primero en hacerlo fue "Tecmed", en cuyo laudo la demanda de expropiación indirecta respectiva fue analizada inicialmente desde la perspectiva de la doctrina del efecto ("Tecmed c/ México", 2003, párr. 113-117). En un giro inesperado, sin embargo, el tribunal decidió hacer uso del principio de proporcionalidad, en los siguientes términos:

\begin{abstract}
El acto o medida no debe imponer una carga o peso excesivo en el inversor extranjero en relación con la finalidad perseguida por el acto reputado como expropiatorio. En la valoración de dicha carga o peso, juega un rol primordial la entidad o dimensión de la privación de propiedad operada por la actuación estatal, y si tal privación fue compensada o no. También debe tenerse en cuenta, en razón de distintos factores legales y prácticos, que el inversor extranjero tiene reducida o ninguna ingerencia [sic] en la toma de decisiones que le afectan, en parte a raíz de su exclusión del ejercicio de
\end{abstract}

\footnotetext{
${ }^{37}$ Este tratado está vigente desde 2004. Ver Asteriti (2013, p. 463-464) y Bücheler (2015, p. 136-138).

${ }^{38}$ E.g. Anexo 11-B del TLC EE.UU.-Korea de 2007; Anexo 13 del TLC China-Nueza Zelanda de 2008; Anexo 2 del Acuerdo Comprehensivo de Inversiones de la ANSA de 2009. Todos estos tratados están vigentes.
} 
derechos políticos reservados a los nacionales del Estado que las adopta, tales como la elección de las autoridades en las que tales decisiones se originan (“Tecmed c/ México", 2003, párr. 118-121).

A pesar de haber declarado que "ese análisis tiene como punto de partida la necesaria deferencia debida al Estado al definir las razones de interés o utilidad pública en que funda su actuación y las formas bajo las que ésta habrá de concretarse”, el tribunal estimó pertinente “juzgar la actuación del Estado [...] sobre la base de su razonabilidad en relación con el fin perseguido, la privación económica causada, y las expectativas legítimas de quién lo sufrió” (“Tecmed c/ México, 2003, párr. 122). Así lo hizo, y concluyó que México había violado el TBI aplicable, por cuanto la medida respectiva se había motivado exclusivamente en consideraciones políticas (“Tecmed c/ México, 2003, párr. 129-132, 139, 144-145, 148). ${ }^{39}$ Con esto, el tribunal llegó al mismo resultado que ya había alcanzado aplicando la doctrina del efecto, pero por otra vía (Bücheler, 2015, p. 144).

En su breve détour argumentativo, "Tecmed" acudió sin mayores explicaciones a la jurisprudencia del TEDH, concretamente a "James" (1986), "Mellacher" (1989), "Pressos Compania Naviera" (1995), y "Matos e Silva" (1996) (Calamita, 2014, pp. 186187) ${ }^{40}$ Estos son casos resueltos por dicho tribunal en aplicación de lo dispuesto en el artículo 1 del Primer Protocolo Opcional del Convenio Europeo para la Protección de los Derechos Humanos y de las Libertades Fundamentales (CEDH). ${ }^{41}$

Tal como ha sido interpretado por el TEDH en numerosas oportunidades, este artículo establece tres reglas: privaciones de la propiedad, controles en su uso, y otras interferencias, como categoría residual. Estas reglas son diferentes, pero se encuentran vinculadas por la aplicación común de dos principios, que interactúan entre sí: el de proporcionalidad y el de margen de apreciación. Por medio del primero, el TEDH busca determinar si hubo un balance justo entre los intereses comunitarios e individuales involucrados en el caso respectivo (Schabas, 2017, pp. 958-985). Este principio debe aplicarse junto al segundo, en virtud del cual este tribunal le reconoce a los Estados parte

\footnotetext{
${ }^{39}$ Ver Calamita (2014, pp. 186-187) y López Escarcena (2014a, pp. 127-129).

${ }^{40}$ Ver "Tecmed c/ México" (2003, párr. 122). Ver en general "James c/ Reino Unido" (1986); "Mellacher c/ Austria" (1989); "Pressos Compania Naviera c/ Bélgica” (1995); "Matos e Silva c/ Portugal” (1996). La jurisprudencia del TEDH fue recuperada de https://www.echr.coe.int/Pages/home.aspx?p=caselaw/HUDOC\&c=

${ }^{41}$ En vigor a partir de 1953.
} 
del CEDH una amplia deferencia en la adopción de medidas regulatorias (AraiTakahashi, 2013). Dado que de las tres reglas que ha reconocido en el artículo 1 del Primer Protocolo Opcional, solo las privaciones de propiedad deben necesariamente ir acompañadas de indemnización, el TEDH cuenta con otras opciones para resolver que hubo un incumplimiento convencional, sin que esto signifique establecer además el pago de una suma de dinero (López Escarcena, 2012). ${ }^{42}$

En el derecho comparado, de donde proviene el principio de proporcionalidad, se entiende que este debe cumplir tres requisitos. ${ }^{43} \mathrm{Al}$ primero de ellos se le conoce como idoneidad, y se refiere a que la medida sea apropiada al objetivo buscado. El segundo, recibe el nombre de necesidad y dice relación con que la medida afecte lo menos posible a la persona involucrada. Al tercero, en tanto, se le llama proporcionalidad stricto sensu $\mathrm{y}$ tiene que ver con que se respete un balance justo o razonable entre los intereses comunitarios e individuales comprometidos (Calamita, 2014, pp. 172-173; Ranjan, 2014, pp. 855-856; Ranjan \& Anand, 2016, pp. 146-147); Vadi, 2014a, p. 204; 2014b, p. 347; 2015, pp. 568-569). ${ }^{44}$ Desde fines de los años 60, el TEDH ha utilizado regularmente este principio, centrando su interpretación y aplicación en el sub-principio de la proporcionalidad stricto sensu (Calamita, 2014, pp. 180-181; López Escarcena, 2013). ${ }^{45}$

En su jurisprudencia sobre el artículo 1 del Primer Protocolo Opcional, el TEDH ha señalado que este busca alcanzar un balance justo entre el interés general y el derecho de propiedad (Bücheler, 2015, p. 134). A pesar de que esta disposición no exige expresamente que se indemnicen las expropiaciones, el TEDH ha entendido que estas deben ir acompañadas del pago de un monto de dinero, el cual no requiere ser el valor de mercado (Perkams, 2010, pp. 111-114). ${ }^{46}$ Para que una expropiación sea legal bajo el

\footnotetext{
${ }^{42}$ Por su parte, el sistema interamericano distingue entre privaciones, que requieren ser indemnizadas, y restricciones o limitaciones a la propiedad, que no dan derecho a indemnización. A diferencia de la jurisprudencia del TEDH, la de la Corte Interamericana de Derechos Humanos (Corte IDH) relativa al artículo 21 de la Convención Americana sobre Derechos Humanos, o Pacto de San José de Costa Rica, no ha influido en los arbitrajes de inversiones. Al respecto, cabe señalar que la Corte IDH no ha aplicado aún el principio de margen de apreciación. Sobre la jurisprudencia de la Corte IDH ver en general López Escarcena (2014b).

43 Además de Alemania, son varios los países en los que se ha desarrollado la doctrina de la proporcionalidad (Bücheler, 2015, pp. 34-50).

Respecto a la migración del principio de proporcionalidad desde el derecho público comparado ver en general Vadi (2014b; 2015).

${ }^{44}$ Ver Asteriti (2013, p. 465) y Kingsbury \& Schill (2010, pp. 85-88). Cf. Bücheler (2015, p. 37) y Henckels (2012, pp. 226-228).

${ }^{45} \mathrm{La}$ Corte IDH ha utilizado el principio de proporcionalidad desde inicios de este siglo y, si bien pareciera distinguir los tres sub-principios doctrinales, en la práctica tiende a restringir su interpretación y aplicación a la proporcionalidad stricto sensu (López Escarcena, 2014b).

${ }^{46}$ Ver Perkams (2010, pp. 114-121).
} 
CEDH, necesita cumplir el test de proporcionalidad entre los medios utilizados y el objetivo que persigue esta (Bücheler, 2015, p. 134).

\section{Una jurisprudencia vacilante}

A la fecha, no son muchos los arbitrajes de inversiones que han hecho mención el principio de proporcionalidad, al momento de resolver demandas de expropiación indirecta (Bücheler, 2015, pp. 130-132; Calamita, 2014, pp. 185-192; Henckels, 2012, pp. 230-237; Kingsbury \& Schill, 2010, pp. 89-96; Vadi, 2014b, pp. 349-350; 2015, pp. 578-579). ${ }^{47}$ En "Azurix" (2006), el tribunal consideró que los argumentos expuestos por "Tecmed" en favor de la utilización de este principio "brindan una orientación útil a los fines de determinar si las medidas reglamentarias serían expropiatorias y darían lugar al pago de una indemnización" (párr. 312). ${ }^{48}$ Sin perjuicio de estas consideraciones, el uso de la proporcionalidad en "Azurix" no pasó de ser una declaración sin mayores consecuencias, dado que el tribunal resolvió la demanda de expropiación indirecta correspondiente aplicando la doctrina del efecto (párr. 322). ${ }^{49}$

En este sentido, la lectura de "LG\&E" (2006) resulta mucho más interesante. Esta decisión judicial señaló que "[p]ara establecer si las medidas estatales resultan en una expropiación en los términos del [TBI aplicable], el Tribunal debe equilibrar dos intereses contrapuestos: el grado de interferencia de la medida con los derechos de propiedad del inversor y el poder del Estado de dictar sus políticas" (“LG\&E c/ Argentina”, 2006, párr. 189). Citando a "Tecmed", el tribunal ahondó en lo que implica este test de balanceo:

\footnotetext{
Con respecto al poder del Estado para dictar sus políticas, se puede decir en general, que el Estado tiene el derecho de dictar medidas con un propósito social o de bienestar general. En este caso, las medidas deben aceptarse sin que sea procedente reclamo alguno por responsabilidad, salvo que se trate de una actuación del Estado manifiestamente desproporcionada con respecto a la necesidad que se trata de abordar. La proporcionalidad con la
}

\footnotetext{
${ }^{47}$ Ver López Escarcena (2014a, p. 129).

${ }^{48}$ Ver "Azurix c/ Argentina” (2006, párr. 311). Ver Vadi (2015, p. 579).

${ }^{49}$ Lo mismo ocurrió en "Fireman`s Fund" (2006, párr. 176-177, 217), que se falló en forma casi simultánea a este caso.
} 
que se debe ejercer este derecho se reconoció en "Tecmed" [...] ("LG\&E c/ Argentina", 2006, párr. 195). ${ }^{50}$

No obstante estas declaraciones, "LG\&E” falló la demanda correspondiente desde la doctrina del efecto, lo que llevó al tribunal a concluir que Argentina no había incumplido el tratado de inversión respectivo (párr. 198-200). ${ }^{51}$ Un par de años después, “Continental Casualty” (2008) partió por señalar que:

hay limitaciones al uso de la propiedad en el interés público que corresponden a regulaciones gubernamentales a la propiedad típicas, que conllevan principalmente limitaciones inevitables impuestas para asegurar los derechos de otros o del público en general (que en último término también son favorables para la propiedad afectada). Estas restricciones no impiden el uso básico, típico de un determinado bien y no imponen una carga irrazonable al dueño, si se le compara con otros propietarios similares. En consecuencia, estas restricciones no se consideran como una forma de expropiación y no requieren de indemnización, siempre que no afecten a la propiedad de una manera intolerable, discriminatoria o desproporcionada (párr. 276).

En apoyo de esta aseveración, el tribunal hizo referencia al artículo 1 del Primer Protocolo Opcional de la CEDH y a "LG\&E", aunque de manera más bien tangencial (“Continental Casualty c/ Argentina”, 2008, párr. 276-277). Como en el caso anterior, “Continental Casualty" finalmente recurrió a la doctrina el efecto para fallar la demanda de expropiación indirecta (párr. 283-285). ${ }^{52}$

Con posterioridad a este laudo, dos casos volvieron a vincular sin mayor coherencia el principio de proporcionalidad con la protección contra la expropiación ilegal que ofrecen los tratados de inversión. En "Total” (2010), el tribunal resolvió parte importante de la demanda respectiva desde la doctrina del efecto, antes de señalar que las

\footnotetext{
${ }^{50}$ Ver "LG\&E c/ Argentina” (2006, párr. 192) y "Tecmed c/ México" (2003, párr. 115).

${ }^{51}$ Ver "LG\&E c. Argentina" (2006, párr. 185-188, 190-191, 193-194, 196-197). Ver Bücheler (2015, p. 130) y Calamita (2014, p. 188-189). Ese mismo año, “Archer Daniels Midland” (2007, párr. 250) incluyó dentro de los factores a considerar para fallar una demanda de expropiación indirecta, el hecho que la medida respectiva sea proporcional o necesaria para un fin legítimo. Como en ocasiones anteriores, el tribunal hizo esta declaración a pesar de haber acogido los postulados de la doctrina del efecto ("Archer Daniels Midland c/ México", 2007, párr. 240-249, 251-252). Corresponde señalar que la traducción al castellano de este laudo no utiliza la palabra "proporcional", sino que "adecuada".

52 Ver Bücheler (2015, p. 130).
} 
medidas estatales de aplicación general, adoptadas de buena fe, y que pueden ser calificadas como razonables en el contexto en el que se dictaron y proporcionadas a los objetivos perseguidos, no serían expropiatorias (párr. 197). ${ }^{53}$

“Tza" (2011), en cambio, aplicó el principio de proporcionalidad a la demanda de expropiación indirecta, sin mencionarlo. En este caso, el tribunal comenzó su análisis desde la doctrina del efecto (párr. 140-149). Sin mayores explicaciones, "Tza" complementó esta aproximación con un singular test, compuesto de cuatro elementos: el impacto y la duración de la medida, en el sentido de interferencia sustancial y permanente; el interés público y la no discriminación, como posibles exenciones de responsabilidad internacional; la eficacia del comportamiento del Estado anfitrión; y la disponibilidad de remedios legales, como condición de debido proceso (párr. 150-240). En virtud de dicho test, el tribunal concluyó que Perú había expropiado ilegalmente el inversionista extranjero, al no haberlo indemnizado; que las medidas estatales fueron injustificadas; que estas tuvieron un resultado abrumadoramente negativo en el demandante; quien no tuvo a su disposición remedios legales adecuados para revertir esta situación ("Tza c/ Perú", 2011, párr. 170, 205, 218-219, 240). ${ }^{54}$

“Occidental" (2012) es otro caso que ofrece consideraciones interesantes sobre el principio de proporcionalidad. Con menciones a "Tecmed" y "Azurix", esta decisión judicial señaló que “[e]n cuanto a la aplicación de la proporcionalidad en forma generalizada en el contexto de los actos administrativos, el cuerpo de jurisprudencia más desarrollado se encuentra en Europa", agregando en seguida que "el Tribunal observa que existe un ordenamiento creciente de normas arbitrales, especialmente en el marco de los arbitrajes ante el CIADI, que establece que el principio de proporcionalidad es aplicable a los posibles incumplimientos de las obligaciones que surgen de los tratados bilaterales de inversión” (“Occidental c/ Ecuador”, 2012, párr. 403, 404). ${ }^{55}$

A pesar de estas aseveraciones, “Occidental” aprovechó que el derecho público ecuatoriano reconoce la proporcionalidad para evitar tener que aplicarla desde el derecho internacional de la inversión, resolviendo la demanda de expropiación indirecta en base a las consideraciones hechas en el laudo sobre la cláusula de trato justo y equitativo del

\footnotetext{
${ }^{53}$ Ver "Total c/ Argentina" (2010, párr. 195-196, 198-199).

${ }^{54}$ Ese mismo año, "El Paso" rechazó la doctrina del poder de policía y optó por la del efecto, haciendo mención al principio de proporcionalidad como accesorio a esta aproximación ("El Paso c/ Argentina" 2011, párr. 233-256).

${ }^{55}$ Ver “Occidental c/ Ecuador” (2012, párr. 402-409, 427).
} 
TBI respectivo (párr. 397, 399-400, 455). ${ }^{56}$ Un par de meses después, en "Burlington" (2012) otro tribunal resolvió que la intervención de Ecuador en la inversión del demandante no constituyó una expropiación, debido a que esta fue "necesaria, adecuada, proporcionada dadas las circunstancias" (párr. 164). ${ }^{57}$ Como en "Tecmed", esta declaración aparentemente vino a complementar la aproximación desde la doctrina de los efectos, que ya había adoptado el tribunal en la misma decisión judicial ("Burlington c/ Ecuador", 2012, párr. 156)..$^{58}$

\section{Un principio migrante}

El derecho internacional de la expropiación reconoce una excepción de poder policía, en virtud de la cual ciertos tipos de intervención estatal no debieran constituir expropiación indirecta, en la medida que no priven de su propiedad al afectado (Mouyal, 2018, pp. 168-169). ${ }^{59} \mathrm{Si}$ bien esta excepción se condice con la redacción de la mayoría de las cláusulas de expropiación condicionada existentes en los tratados de inversión actualmente en vigor, no ocurre así con la llamada doctrina del poder de policía, tal como fue interpretada y aplicada en "Methanex" y en otros arbitrajes de inversiones. ${ }^{60}$

El problema con esta aproximación radica en que toda privación de propiedad que haga un Estado puede ser considerada, de una u otra manera, por causa de utilidad pública. Descartar la naturaleza expropiatoria de una medida regulatoria por esta simple razón, transforma en superflua una condición de larga data en el derecho internacional de la expropiación, que contemplan virtualmente todos los tratados de inversión (Bücheler, 2015, pp. 128-129; De Nanteuil, 2017, pp. 12-13; Mouyal, 2018, pp. 176-178, 188; Ranjan, 2014, pp. 868-872; Ranjan \& Anand, 2016, pp. 137-138, 139). ${ }^{61}$

\footnotetext{
56 Ver Calamita (2013, pp. 189-192).

57 Ver "Burlington c/ Ecuador" (2012, párr. 152-153, 170). Ver Vadi (2014b, pp. 349-350; 2015, p. 579).

58 Ver "Burlington c/ Ecuador" (2012, párr. 170). Con posterioridad a este laudo, el principio de proporcionalidad volvió a ser aludido en "Urbaser" y "Philip Morris", aun cuando en ambos laudos el tribunal respectivo ya había adoptado una aproximación favorable a la doctrina del poder de policía (“Urbaser c/ Argentina", 2016, párr. 953-954, 956, 965-966, 969, 974, 976, 978, 980-983, 985-992; "Philip Morris c/ Uruguay”, 2016, párr. 286-287, 290-301, 305).

${ }^{59}$ Ver Ranjan \& Anand (2016, p. 141).

${ }^{60}$ Ver "Methanex c/ EE.UU." (2005, párr. 7). Algo similar a lo declarado por este tribunal respecto a las expropiaciones indirectas puede leerse, por ejemplo, en "Chemtura c/ Canadá" (2010, párr. 266), "El Paso c/ Argentina" (2011, párr. 240), "Saluka c/ República Checa” (2006, párr. 262), "Philip Morris c/ Uruguay" (2016, párr. 290-301) y “Urbaser c/ Argentina” (2016, párr. 986, 989-991). Ver de Nanteuil (2017, pp. 1213, 15-16) y Mouyal (2018, pp. 190-191).

${ }^{61}$ Ver Mostafa (2008, pp. 272-273, 277-278, 287, 295). Cf. Henckels (2012, nota 5).
} 
Esta solución, hace que un requisito de legalidad expropiatoria mude radicalmente en causal de exención de responsabilidad internacional, en base a argumentaciones judiciales desprovistas de base convencional.

A fin de superar los manifiestos inconvenientes que presenta la doctrina del poder de policía, se ha propuesto la aplicación de la proporcionalidad a las demandas de expropiación indirecta como una especie de versión mitigada de dicha teoría (Asteriti, 2013, p. 469; Bücheler, 2015, pp. 129-132, 153, 156, 177; De Nanteuil, 2017, pp. 11-14; Mouyal, 2018, pp. 190-193). ${ }^{62}$ Recurriendo a la jurisprudencia del TEDH, algunos tribunales en arbitrajes de inversiones han empleado este principio para salir de las respuestas de tipo todo o nada que ofrecen las doctrinas antagónicas del efecto y del poder de policía. Como suele ocurrir con los trasplantes jurídicos, diferencias en su implementación han terminado por deformar el sentido que tiene la proporcionalidad en su jurisdicción de origen. ${ }^{63}$

Para explicar esta situación, es necesario ir al artículo 14 de Ley Fundamental de la República Federal Alemana, y revisar la primera encarnación judicial que tuvo este principio, en relación al derecho de propiedad (Asteriti, 2013, p. 465; Calamita, 2014, nota 81; Kingsbury \& Schill, 2010, pp. 79-81; Ranjan, 2014, pp. 855-856) ${ }^{64}$ Es en la parte final de esta disposición donde se puede apreciar la principal diferencia que tiene con sus equivalentes del Primer Protocolo Opcional de la CEDH, y de los TBI y los capítulos de inversión de los AIE. Al tener que considerar en forma equitativa los intereses de la comunidad y de los afectados, el artículo 14 de la Ley Fundamental está haciendo una clara alusión al principio de proporcionalidad, lo que no ocurre con las otras disposiciones. Para el Tribunal Constitucional Federal Alemán (TCFA), la noción de expropiación es muy limitada, pues requiere afectar a personas determinadas e ir acompañada de una apropiación estatal (Perkams, 2010, p. 134). Es por esta razón, que las medidas regulatorias de carácter general contempladas en los dos primeros numerales del artículo 14 de la Ley Fundamental, incluso aquellas que interfieren considerablemente con la propiedad privada, no son necesariamente expropiatorias en Alemania (Bücheler, 2015, p. 133; Perkams, 2010, pp. 134-135).

\footnotetext{
${ }^{62}$ Ver Kingsbury \& Schill (2010, pp. 77-79, 88-89). Ver en general Kriebaum (2007).

${ }^{63}$ Sobre los trasplantes jurídicos ver en general Bonilla (2009).

${ }^{64}$ Ver Bucheler (2015, pp. 34-35, 42, 132-134) y Perkams (2010, pp. 130-137). Cf. Henckels (2012, pp. 226-227). La Ley Fundamental de la República Federal Alemana fue recuperada de https://www.btgbestellservice.de/pdf/80206000.pdf.
} 
Esto ya es una diferencia importante con la protección contra la expropiación que ofrecen los tratados de inversión. Pero hay más. En Alemania, el análisis de constitucionalidad de la medida estatal en cuestión, donde se emplea el principio de proporcionalidad, se da en un contexto muy distinto al de los arbitrajes de inversiones. Esto, por cuanto conforme al artículo 14 de la Ley Fundamental el Estado alemán no debe pagar una indemnización íntegra por la pérdida sufrida, sino una que refleje un balance entre los intereses comunitarios e individuales involucrados. En otras palabras, entre el interés público buscado y el impacto que tiene la expropiación o la medida regulatoria de carácter general en el propietario afectado. Aplicando este test, bien puede resultar que el monto de la indemnización correspondiente sea inferior al valor de mercado del bien expropiado (Bücheler, 2015, p. 133; Perkams, 2010, p. 135-137).

Los TBI y los capítulos de inversión de los AIE de primera generación no le ofrecen esta flexibilidad a los tribunales que deciden casos de expropiación indirecta en arbitrajes de inversiones. Violándose el artículo correspondiente, el Estado deberá pagar una indemnización pronta, adecuada y efectiva. Es por esto que Noruega propuso en su TBI modelo de 2007 replicar, en esencia, el artículo 1 del Primer Protocolo Opcional (TBI modelo de Noruega, 2007, Art. 6). ${ }^{65}$ Abiertamente reconociendo que la nueva cláusula provino de dicha disposición, los comentarios a este TBI modelo no ocultaron que uno de sus objetivos era que los inversionistas extranjeros en Noruega tengan una protección contra la expropiación que no sea superior a la que ese Estado ofrece a sus propios nacionales. ${ }^{66}$ En 2009, este país abandonó el modelo del año anterior, reemplazándolo en 2015 por otro que retuvo la redacción de esta disposición. Sin embargo, este nuevo modelo también ha sido aparentemente abandonado, pues Noruega no ha vuelto a celebrar un TBI desde 1996 (Bücheler, 2015, p. 136, 139-140).

Estos modelos noruegos fueron los primeros en hacerse cargo del primer gran problema que tiene la aplicación del principio de proporcionalidad a las cláusulas de expropiación condicionada de los tratados de inversión: su falta de soporte convencional. Los nuevos tratados de inversión de EE.UU., Canadá y otros Estados, actualmente en vigencia, han incluido clarificaciones que le permitirían a los tribunales internacionales

\footnotetext{
${ }^{65}$ Recuperado de https://www.italaw.com/sites/default/files/archive/ita1031.pdf

66 Los comentarios al TBI modelo de Noruega de 2007 están disponibles en https://www.italaw.com/sites/default/files/archive/ita1029.pdf Estos comentarios incluso señalaron que el valor de mercado excede la obligación de indemnizar una expropiación que contemplan la jurisprudencia del TEDH y el derecho noruego.
} 
interpretar la clásica excepción del poder de policía de una manera amplia, restringiendo así las medidas estatales que eventualmente puedan llegar a constituir una expropiación. $\mathrm{Si}$ bien estos acuerdos internacionales no introducen de manera explícita la proporcionalidad en sus articulados, se podría entender que dicho principio está implícitamente incorporado en dos de los tres elementos de análisis que incluyen para determinar la naturaleza expropiatoria de una medida: el impacto que esta tiene en el propietario, y su carácter. O sea, su intención (Bücheler, 2015, pp. 136-137; De Nanteuil, 2017, pp. 17-21). Esto vendría a solucionar uno de los problemas que presentó la aplicación de la proporcionalidad en "Tecmed", donde a pesar de no contar con apoyo en el TBI respectivo, el tribunal utilizó dicho principio para determinar la naturaleza expropiatoria de la medida regulatoria adoptada por México. En el análisis judicial respectivo, la utilidad pública jugó un papel central.

De esta manera, la aproximación de "Tecmed" adoleció del mismo inconveniente que tiene la doctrina del poder de policía tradicional: transformó un requisito convencional de legalidad expropiatoria en un eximente jurisprudencial de responsabilidad internacional (Bücheler, 2015, p. 144). La doctrina del poder de policía mitigada, que ofrecen los articulados de estos nuevos tratados de inversión, vendría a solucionar esta dificultad (Bücheler, 2015, pp. 153-157; Kingsbury \& Schill, 2010, pp. 91-92).

Sin embargo, con esta alternativa no se supera el otro problema que presenta la línea jurisprudencial que comienza en "Tecmed". En los arbitrajes de inversiones, la proporcionalidad se utiliza al momento de establecer la existencia de una expropiación indirecta. El TEDH, en cambio, emplea este principio para determinar la legalidad de la medida estatal correspondiente, en cuyo análisis el pago de una indemnización es un factor importante (Kriebaum, 2007, p. 730; Ruiz Fabri, 2003, pp. 155-157, 163-165). ${ }^{67}$ Con esto, la proporcionalidad cumple una función correctiva en Europa. La aplicación de dicho principio en un momento u otro produce resultados contrapuestos, ya que mientras en los nuevos tratados de inversión se busca favorecer al Estado anfitrión, en la jurisprudencia del TEDH el objetivo es otorgar una protección adicional al propietario afectado (Bücheler, 2015, pp. 146-148, 150-151, 152-153). Esta diferencia no es menor, pues mientras la utilización que han hecho algunos arbitrajes de inversiones del principio

${ }^{67}$ Ver Schabas (2017, pp. 976-977). 
de proporcionalidad desvirtúa su sentido primigenio, la del TEDH se ajusta bien a este (Vadi, 2014a, p. 208; 2014b, p. 355; 2015, p. 573).

\section{Conclusión}

La extensa red de tratados de inversión que existe en la actualidad contiene una protección contra la expropiación ilegal, tanto directa como indirecta. En la determinación de cuándo una medida regulatoria pasa a tener una naturaleza expropiatoria, la doctrina y la jurisprudencia internacional generalmente han optado por dos posturas antagónicas, que privilegian el efecto de la providencia respectiva, por un lado, o la intención de esta, por otro. Mientras los Estados en vías de desarrollo eran los únicos demandados en los arbitrajes de inversiones, pocos advirtieron algún inconveniente en que los tribunales prefieran la doctrina del efecto por sobre la del poder de policía.

Cuando las demandas interpuestas en virtud del Capítulo 11 del NAFTA comenzaron a recaer en todos los Estados parte de este acuerdo internacional, y no solo en México, esto cambió drásticamente. EE.UU. modificó su política de inversiones extranjeras y comenzó a reemplazar sus tratados de inversión por otros, que incluyen clarificaciones a sus cláusulas de expropiación condicionada, destinadas a facilitar la aplicación de la doctrina del poder de policía en eventuales arbitrajes de inversiones. Casi en forma paralela a esta evolución convencional, otra posible solución empezó a surgir jurisprudencialmente en el derecho internacional de la inversión. Esta procede del sistema europeo de derechos humanos, y consiste en la aplicación del principio de proporcionalidad al establecimiento del carácter expropiatorio de las medidas estatales impugnadas judicialmente.

No obstante, los tratados de inversión no contemplan las tres reglas que ha identificado el TEDH en el artículo 1 del Primer Protocolo Opcional de la CEDH. Este inconveniente, pareciera haberse superado con las clarificaciones incorporadas a los TBI y capítulos de inversión de los AIE de nueva generación. Sin embargo, persiste otro problema. A diferencia del artículo 14 de la Ley Fundamental de la República Federal Alemana, el artículo 1 del Primer Protocolo Opcional de la CEDH no menciona al principio de proporcionalidad. Ha sido la jurisprudencia del TEDH quien lo ha hecho aplicable a esta disposición, utilizándolo al momento de establecer la legalidad de la 
medida estatal respectiva, que es una etapa posterior a la determinación de su naturaleza. Los tratados de inversión de nueva generación tampoco mencionan expresamente a la proporcionalidad en sus articulados. Pero a diferencia de lo que ocurre en el sistema europeo de derechos humanos, en los arbitrajes de inversiones se recurre a este principio al momento de establecer el carácter expropiatorio de la medida en cuestión. Esta aplicación de la proporcionalidad a etapas distintas del análisis judicial correspondiente produce resultados contrapuestos en la jurisprudencia del TEDH y en los arbitrajes de inversiones. No es de extrañar, puesto que la utilización de este principio tiene distintos objetivos en ambos foros: favorecer al propietario afectado, en el primero; y entregar una protección adicional al Estado anfitrión, en los segundos.

En tanto no se supere este problema, la confusa aplicación de la proporcionalidad en los arbitrajes de inversiones no podrá alzarse como una alternativa viable para resolver demandas de expropiación indirecta, incluso en aquellos casos en que se requiera interpretar una cláusula de nueva generación. Empleado correctamente, junto al margen de apreciación, este principio podría constituir un instrumento útil para encontrar soluciones adecuadas para este tipo de casos. No obstante, esto requería de una clara base normativa, similar a la que le ha permitido al TEDH desarrollar su jurisprudencia sobre la protección de la propiedad, que por el momento no existe en el derecho internacional de la inversión.

\section{Financiamiento}

Este trabajo es parte del proyecto Fondecyt Regular No 1161185 , adjudicado el 2016 y titulado "El empleo del test de proporcionalidad en las expropiaciones indirectas y en otras injerencias no privativas del derecho de propiedad".

\section{Referencias}

Arai-Takahashi, Y. (2013). The Margin of Appreciation Doctrine: A Theoretical Analysis of Strasbourg's Variable Geometry. En A. Føllesdal, B. Peters, \& G. Ulfstein (Eds.), Constituting Europe: The European Court of Human Rights in a National, European and Global Context (pp. 62-105). Cambridge: CUP.

Asteriti, A. (2013). Regulatory Expropriation Claims in International Investment Arbitration: A Bridge Too Far? En A. Bjorklund (Ed.), Yearbook on International Investment Law and Policy 2012-2013 (pp. 451-473). Oxford: OUP. 
Bonilla, D. (Ed.). (2009). Teoría del derecho y trasplantes jurídicos. Bogotá, Colombia: Uniandes.

Bücheler, G. (2015). Proportionality in Investor-State Arbitration. Oxford: OUP.

Calamita, N. J. (2014). The Principle of Proportionality and the Problem of Indeterminacy in International Investment Treaties. En A. Bjorklund (Ed.), Yearbook on International Investment Law and Policy 2013-2014 (pp. 157-200). Oxford: OUP.

De Nanteuil, A. (2017). Recent Developments in Expropriation Law: Towards a Better Protection of States' Sovereign Interests? En T. El Ghadban, C-M. Mazuy, \& A. Senegacnik (Eds.), La protection des investissements étrangers: vers une réaffirmation de l'état? / The Protection of Foreign Investments: A Reaffirmation of the State? (pp. 922). París, Francia: Pedone.

Dolzer, R., \& Stevens, M. (1995). Bilateral Investment Treaties. La Haya, Países Bajos: Martinus Nijhoff.

Duggal, K. A. N., García Clavijo, D. F., Trujillo, S., \& Rincón, M. C. (2019). Colombia’s 2017 Model IIA: Something Old, Something New, Something Borrowed. ICSID Review Foreign Investment Law Journal, 34(1), 224-240.

Durney, M. (2013). Legal Effects and Implications of the Denunciation of the ICSID Convention on Unilateral Consent Contained in Bilateral Investment Treaties: A Perspective from Latin American Cases. En A. von Bogdandy, A. Peters, \& R. Wolfrum (Eds.), Max Planck Yearbook of United Nations Law (pp. 221-304). Leiden, Holanda: Martinus Nijhoff.

Fortier, L. Y. (2009). The Canadian Approach to Investment Protection: How Far We Have Come! En C. Binder; U. Kriebaum, A. Reinisch, \& S. Wittich (Eds.), International Investment Law for the 21st Century: Essays in Honour of Christoph Schreuer (pp. 525543). Oxford: OUP.

Henckels, C. (2012). Indirect Expropriation and the Right to Regulate: Revisiting Proportionality Analysis and the Standard of Review in Investor-State Arbitration. Journal of International Economic Law, 15(1), 223-255.

Kingsbury, B., \& Schill, S. W. (2010). Public Law Concepts to Balance Investors' Rights with State Regulatory Actions in the Public Interest - the Concept of Proportionality. En S. W. Schill (Ed.), International Investment Law and Comparative Public Law (pp. 75104). Oxford: OUP.

Kriebaum, U. (2007). Regulatory Takings: Balancing the Interests of the Investor and the State. Journal of World Investment \& Trade, 8(5), 717-744.

Kulick, A. (Ed.). (2018). Reassertion of Control over the Investment Treaty Regime. Cambridge: CUP.

Legarre, S. (2007). The Historical Background of the Police Power. University of Pennsylvania Journal of Constitutional Law, 9(3), 745-796.

Lim, C. L. (2016). The Worm's View of History and the Twailing Machine. En C. L. Lim (Ed.), Alternative Visions of the International Law on Foreign Investment: Essays in Honour of Muthucumaraswamy Sornarajah (pp. 3-42). Oxford: OUP. 
López Escarcena, S. (2012). Interferences with Property under European Human Rights Law. Florida Journal of International Law, 24(3), 513-544.

López Escarcena, S. (2013). Expropriations and Other Measures Affecting Property Rights in the Case Law of the Iran-United States Claims Tribunal. Wisconsin International Law Journal, 31(2), 176-207.

López Escarcena, S. (2014a). Indirect Expropriation in International Law. Cheltenham: Edward Elgar.

López Escarcena, S. (2014b). La propiedad y su privación o restricción en la jurisprudencia de la Corte Interamericana. Ius et Praxis, 20(2), 531-576.

Lusa Bordin, F. (2018). Reasserting Control through Withdrawal from Investment Agreements: What Role for the Law of Treaties? En A. Kulick (Ed.), Reassertion of Control over the Investment Treaty Regime (pp. 209-229). Cambridge: CUP.

Mostafa, B. (2008). The Sole Effects Doctrine, Police Powers and Indirect Expropriation under International Law. Australian International Law Journal, 15, 265-296.

Mouyal, L. W. (2018). International Investment Law and the Right to Regulate: A Human Rights Perspective. Nueva York, NY: Routledge.

Perkams, M. (2010). The Concept of Indirect Expropriation in Comparative Public Law Searching for Light in the Dark. En S. W. Schill (Ed.), International Investment Law and Comparative Public Law (pp. 107-150). Oxford: OUP.

Plater, Z. J., Abrams, R. H., Graham, R. L., Heinzerling, L., Wirth, D. A., \& Hall, N D. (2010). Environmental Law and Policy: Nature Law and Society. Nueva York, NY: Wolters Kluwer.

Ranjan, P. (2014). Using the Public Law Concept of Proportionality to Balance Investment Protection with Regulation in International Investment Law: A Critical Appraisal. Cambridge Journal of International and Comparative Law, 3(3), 853-883.

Ranjan, P., \& Anand, P. (2016). Determination of Indirect Expropriation and Doctrine of Police Power in International Investment Law: A Critical Appraisal. En L. Choukrone (Ed.), Judging the State in International Trade and Investment Law: Sovereignty Modern, the Law and the Economics (pp. 127-151). Ciudad de Singapur, Singapur: Springer.

Ruiz Fabri, H. (2003). The Approach taken by the European Court of Human Rights to the Assessment of Compensation for Regulatory Expropriations of the Property of Foreign Investors. New York University Environmental Law Journal, 1(1), 148-173.

Ruzza, A. (2017). Expropriation and Nationalization. En R. Wolfrum (Ed.), Max Planck Encyclopedia of Public International Law. Oxford: OUP.

Sacerdoti, G. (2015). New International Economic Order (NIEO). En R. Wolfrum (Ed.), Max Planck Encyclopedia of Public International Law. Oxford: OUP.

Salacuse, J. W. (2015). The Law of International Investment Treaties. Oxford: OUP. 
Schabas, W. A. (2017). The European Convention on Human Rights: A Commentary. Oxford: OUP.

Stern, B. (2008). In Search of the Frontiers of Indirect Expropriation. En A. W. Rovine (Ed.), Contemporary Issues in International Arbitration and Mediation: The Fordham Papers 2007 (pp. 29-52). Leiden: Martinus Nijhoff.

Titi, C. (2015). International Investment Law and the European Union: Towards a New Generation of International Investment Agreements. European Journal of International Law, 26(3), 639-661.

UNCTAD. (2020a). Investor-State Dispute Settlement Cases Pass the 1,000 Mark: Cases and Outcomes in 2019. Nueva York, NY: ONU.

UNCTAD. (2020b). World Investment Report 2020. Nueva York, NY: ONU.

Vadi, V. (2014a). Proportionality, Reasonableness and Standards of Review in Investment Treaty Arbitration. En A. K. Bjorklund (Ed.), Yearbook on International Investment Law and Policy 2013-2014 (pp. 201-228). Oxford: OUP.

Vadi, V. (2014b). The Migration of Constitutional Ideas: The Strange Case of Proportionality in International Investment Law and Arbitration. En A. K. Bjorklund (Ed.), Yearbook on International Investment Law and Policy 2013-2014 (pp. 337-360). Oxford: OUP.

Vadi, V. (2015). The Migration of Constitutional Ideas to Regional and International Economic Law: The Case of Proportionality. Northwestern Journal of International Law \& Business, 35(3), 557-589.

Vandevelde, K. J. (2009a). A Comparison of the 2004 and 1994 US Model BITs: Rebalancing Investor and Host Country Interests. En K. P. Sauvant (Ed.), Yearbook on International Investment Law and Policy 2008-2009 (pp. 283-315). Oxford: OUP.

Vandevelde, K. J. (2009b). U.S. International Investment Agreements. Oxford: OUP.

Vandevelde, K. J. (2010). Bilateral Investment Treaties: History, Policy, and Interpretation. Oxford: OUP.

Vandevelde, K. J. (2017). The First Bilateral Investment Treaties: U.S. Postwar Friendship, Commerce, and Navigation Treaties. Oxford: OUP.

Von Walter, A., \& Andrisani, M. L. (2019). Resolution of Investment Disputes. En M. M. Mbengue, \& S. Schacherer (Eds.), Foreign Investment Under the Comprehensive Economic and Trade Agreement (CETA) (pp. 185-206). Cham, Suiza: Springer.

Yannaca-Small, K. (Ed.). (2018). Arbitration under International Investment Agreements: A Guide to the Key Issues. Oxford: OUP.

Waibel, M., Kaushal, A., Chung, K-H. L., \& Balchin, C. (Eds.). (2010). The Backlash Against Investment Arbitration. Perceptions and Reality. Alphen aan den Rijn, Países Bajos: Kluwer.

Wei, S. (2015). Expropriation in Transition: Evolving Chinese Investment Treaty Practices in Local and Global Contexts. Leiden Journal of International Law, 28(3), 579-604. 


\section{Jurisprudencia}

"Archer Daniels Midland Company y Tate \& Lyle Ingredients Americas, Inc. c/ México", caso CIADI No. ARB (AF)/04/5 (Laudo, 21 de noviembre de 2007).

“Asian Agricultural Products Ltd. c/ Sri Lanka", caso CIADI No. ARB/87/3 (Laudo, 27 de junio de 1990).

“Azurix Corp. c/ Argentina”, caso CIADI No. ARB/01/12 (Laudo, 14 de julio de 2006).

"Burlington Resources Inc. c/ Ecuador", caso CIADI No. ARB/08/5 (Decisión sobre responsabilidad, 14 de diciembre de 2012).

“Chemtura Corporation c/ Canadá”, arbitraje CNUDMI (Laudo, 2 de agosto de 2010).

“Oscar Chinn (Reino Unido c/ Bélgica)”, CPJI (Sentencia, 12 de diciembre de 1934).

"Continental Casualty Company c/ Argentina", caso CIADI No. ARB/03/9 (Laudo, 5 de septiembre de 2008).

"El Paso Energy International Company c/ Argentina", caso CIADI No. ARB/03/15 (Laudo, 31 de octubre de 2011).

"Fireman's Fund Insurance Company c/ México", caso CIADI No. ARB(AF)/02/1 (Laudo, 17 de julio de 2006).

“James y otros c/ Reino Unido”, TEDH (Sentencia, 21 de febrero de 1986).

"LG\&E Energy Corp., LG\&E Capital Corp., y LG\&E International, Inc. c/ Argentina", caso CIADI No. ARB/02/1 (Decisión sobre responsabilidad, 3 de octubre de 2006).

“Matos e Silva Lda. y otros c/ Portugal", TEDH, (Sentencia, 16 de septiembre de 1996).

"Mellacher y otros c/ Austria", TEDH (Sentencia, 19 de diciembre de 1989).

"Metalclad Corporation c/ México", caso CIADI No. ARB(AF)/97/1 (Laudo, 30 de agosto de 2000).

“Methanex Corporation c/ EE.UU.”, arbitraje CNUDMI (Laudo, Parte IV, Capítulo D, 3 de agosto de 2005).

"Navieros Noruegos (Noruega c/ EE.UU.)", Anderson, Vogt \& Valloton, árbitros (Laudo, 13 de octubre de 1922).

"Occidental Petroleum Corporation y Occidental Exploration and Production Company c/ Ecuador", caso CIADI No. ARB/06/11 (Laudo, 5 de octubre de 2012).

"Penn Central Transportation Co. c/ Ciudad de Nueva York", Corte Suprema de EE.UU. (Sentencia, 26 de junio de 1978).

"Pennsylvania Coal Co. c/ Mahon", Corte Suprema de EE.UU. (Sentencia, 11 de diciembre de 1922). 
"Philip Morris Brands Sàrl, Philip Morris Products S.A. y Abal Hermanos S.A. c/ Uruguay", caso CIADI No. ARB/10/7 (Laudo, 8 de julio de 2016).

"Pressos Compania Naviera S.A. y otros c/ Bélgica", TEDH (Sentencia, 20 de noviembre de 1995).

"Saluka Investments B.V. c/ República Checa", arbitraje CNUDMI (Laudo parcial, 17 de marzo de 2006).

"Sea-Land Service, Inc. c/ República Islámica de Irán y la Organización Portuaria y Naviera", Tribunal de Reclamaciones Irán-EE.UU. (Laudo, 22 de junio de 1984).

"Técnicas Medioambientales Tecmed, S.A. c/ México", caso CIADI No. ARB (AF)/00/2 (Laudo, 20 de mayo de 2003).

"Tippets, Abbett, McCarthy, Stratton c/ TAMS-AFFA Consulting Engineers of Iran, República Islámica de Irán et al.”, Tribunal de Reclamaciones Irán-EE.UU. (Laudo, 29 de junio de 1984).

"Total S.A. c/ Argentina", caso CIADI No. ARB/04/01 (Decisión sobre responsabilidad, 27 de diciembre de 2010).

“Tza Yap Shum c/ Perú”, caso CIADI No. ARB/07/6 (Laudo, 7 de julio de 2011).

"Urbaser S.A. y Consorcio de Aguas Bilbao Bizkaia, Bilbao Biskaia Ur Partzuergoa c/ Argentina", caso CIADI No. ARB/07/26 (Laudo, 8 de diciembre de 2016).

\section{Contribución autoral}

a) Concepción y diseño del trabajo; b) Adquisición de datos; c) Análisis e interpretación de datos; d) Redacción del manuscrito; e) revisión crítica del manuscrito.

S. L. E. ha contribuido en a, b, c, d, e.

\section{Editor científico responsable}

Dr. Pablo Rodríguez Almada 